\title{
Radial growth of the derivatives of analytic functions in Besov spaces
}

\author{
Dedicated to the memory of Peter L. Duren
}

https://doi.org/10.1515/conop-2020-0107

Received August 13, 2020; accepted October 7, 2020

Abstract: For $1<p<\infty$, the Besov space $B^{p}$ consists of those functions $f$ which are analytic in the unit disc $\mathbb{D}=\{z \in \mathbb{C}:|z|<1\}$ and satisfy $\int_{\mathbb{D}}\left(1-|z|^{2}\right)^{p-2}\left|f^{\prime}(z)\right|^{p} d A(z)<\infty$. The space $B^{2}$ reduces to the classical Dirichlet space $\mathcal{D}$. It is known that if $f \in \mathcal{D}$ then $\left|f^{\prime}\left(r e^{i \theta}\right)\right|=\mathrm{o}\left[(1-r)^{-1 / 2}\right]$, for almost every $\theta \in[0,2 \pi]$. Hallenbeck and Samotij proved that this result is sharp in a very strong sense.

We obtain substitutes of the above results valid for the spaces $B^{p}(1<p<\infty)$ an we give also an application of our them to questions concerning multipliers between Besov spaces.

Keywords: Besov spaces, radial behaviour, multipliers

MSC: $30 \mathrm{H} 25,47 \mathrm{~B} 38$

\section{Introduction}

Let $\mathbb{D}=\{z \in \mathbb{C}:|z|<1\}$ denote the open unit disc in the complex plane $\mathbb{C}$ and let $\mathcal{H}$ ol $(\mathbb{D})$ be the space of all analytic functions in $\mathbb{D}$. Also, $d A$ will denote the normalized area measure on $\mathbb{D}: d A(z)=\frac{1}{\pi} d x d y=$ $\frac{1}{\pi} r d r d \theta$. For $0 \leq r<1$ and $f$ analytic in $\mathbb{D}$ we set

$$
\begin{aligned}
M_{p}(r, f) & =\left(\frac{1}{2 \pi} \int_{0}^{2 \pi}\left|f\left(r e^{i \theta}\right)\right|^{p} d \theta\right)^{1 / p}, \quad 0<p<\infty, \\
M_{\infty}(r, g) & =\max _{|z|=r}|f(z)| .
\end{aligned}
$$

For $0<p \leq \infty$ the Hardy space $H^{p}$ consists of those functions $f$, analytic in $\mathbb{D}$, for which

$$
\|f\|_{H^{p}} \stackrel{\text { def }}{=} \sup _{0<r<1} M_{p}(r, f)<\infty .
$$

We refer to [8] for the theory of Hardy spaces. The weighted Bergman space $A_{\alpha}^{p}(0<p<\infty, \alpha>-1)$ consists of those $f \in \mathcal{H} \operatorname{col}(\mathbb{D})$ such that

$$
\|f\|_{A_{\alpha}^{p}} \stackrel{\text { def }}{=}\left((\alpha+1) \int_{\mathbb{D}}\left(1-|z|^{2}\right)^{\alpha}|f(z)|^{p} d A(z)\right)^{1 / p}<\infty .
$$

The unweighted Bergman space $A_{0}^{p}$ is simply denoted by $A^{p}$. We refer to $[9,20,30]$ for the notation and results about Bergman spaces.

Salvador Domınguez: Análisis Matemático, Facultad de Ciencias, Universidad de Málaga, 29071 Málaga, Spain; E-mail: sdmolina16@hotmail.com

*Corresponding Author: Daniel Girela: Análisis Matemático, Facultad de Ciencias, Universidad de Málaga, 29071 Málaga, Spain; E-mail: girela@uma.es 
For $1<p<\infty$, the analytic Besov space $B^{p}$ is defined as the set of all functions $f$ analytic in $\mathbb{D}$ such that $f^{\prime} \in A_{p-2}^{p}$. Thus a function $f \in \mathcal{H} \operatorname{Col}(\mathbb{D})$ belongs to $B^{p}$ if and only if $\rho_{p}(f)<\infty$, where

$$
\rho_{p}(f)=\left\|f^{\prime}\right\|_{A_{p-2}^{p}}=\left((p-1) \int_{\mathbb{D}}\left(1-|z|^{2}\right)^{p-2}\left|f^{\prime}(z)\right|^{p} d A(z)\right)^{1 / p} .
$$

All $B^{p}$ spaces $(1<p<\infty)$ are conformally invariant with respect to the semi-norm $\rho_{B^{p}}$ defined by

$$
\rho_{B^{p}}(f) \stackrel{\text { def }}{=}\left\|f^{\prime}\right\|_{A_{p-2}^{p}}
$$

(see [3, p. 112] or [6, p. 46]) and Banach spaces with the norm $\|\cdot\|_{B^{p}}$ defined by $\|f\|_{B^{p}}=|f(0)|+\rho_{B^{p}}(f)$. The space $B^{1}$ requires a special definition: it is the space of all analytic functions $f$ in $\mathbb{D}$ for which $f^{\prime \prime} \in A^{1}$. Although the corresponding semi-norm is not conformally invariant, the space itself is. Another possible definition (with a conformally invariant semi-norm) is given in [3], where $B^{1}$ was denoted by $\mathcal{M}$. It is well known that the spaces $B^{p}$ form and ascending chain and that they are all contained in the space $B M O A$ [14] and, hence, in the Bloch space $\mathcal{B}[2]$

$$
B^{q} \subsetneq B^{p} \subsetneq B M O A \subsetneq \mathcal{B}, \quad 1 \leq q<p<\infty .
$$

An important and well-studied case is the classical Dirichlet space $B^{2}$, usually denoted by $\mathcal{D}$, of analytic functions whose image has a finite area, counting multiplicities. We mention $[3,6,7,21,22,29,30]$ as references to find a lot of information on Besov spaces.

Obtaining results about the behaviour along radii of distinct classes of analytic functions in the unit disc has shown to be an important question in complex analysis with applications to a good number of problems over the years. One of the best known results in this line is due to Rudin [25] who showed the existence of an $H^{\infty}$-function $f$ for which the radial variation

$$
V(f, \theta)=\int_{0}^{1}\left|f^{\prime}\left(r e^{i \theta}\right)\right| d r
$$

is infinite for every $\theta$ except possibly for those $\theta$ in a set of the first category and of measure zero.

By the definition, it is clear that, for $f \in \mathcal{H} o l(\mathbb{D})$ and $1<p<\infty$, the following two assertions hold:

$$
\begin{gathered}
f \in B^{p} \Leftrightarrow \int_{0}^{1}(1-r)^{p-2} M_{p}\left(r, f^{\prime}\right)^{p} d r<\infty . \\
\text { If } f \in B^{p} \text {, then } \int_{0}^{1}(1-r)^{p-2}\left|f^{\prime}\left(r e^{i \theta}\right)\right|^{p} d r<\infty, \text { for almost every } \theta \in[0,2 \pi] .
\end{gathered}
$$

The authors of this paper have recently proved that (1.2) and (1.3) are sharp in a very strong sense connecting this with (1.1). Indeed, for $1<q<p<\infty$ they have constructed a function $f \in B^{p} \cap H^{\infty}$ with $M_{p}\left(r, f^{\prime}\right)$ "as big as possible" and having "bad integrability properties of order $q$ along all the radii". To be precise, they have proved the following result in [5, Theorem 1].

Theorem A. Suppose that $1<q<p<\infty$ and let $\phi$ be a positive increasing function defined in $[0,1)$ satisfying

$$
\int_{0}^{1}(1-r)^{p-2} \phi(r)^{p} d r<\infty
$$

and

$$
\int_{0}^{1}(1-r)^{q-2} \phi(r)^{q} d r=\infty
$$


Then there exists a function $f \in\left(B^{p} \cap H^{\infty}\right) \backslash B^{q}$ with the following two properties:

$$
\begin{gathered}
M_{p}\left(r, f^{\prime}\right) \gtrsim \phi(r) . \\
\int_{0}^{1}(1-r)^{q-2}\left|f^{\prime}\left(r e^{i \theta}\right)\right|^{q} d r=\infty, \quad \text { for every } \theta \in[0,2 \pi] .
\end{gathered}
$$

Theorem A was applied in [5] to obtain results on multipliers and superposition operators acting on Besov spaces.

Our aim in this work is to obtain sharp estimates on the growth of the derivatives of $B^{p}$-functions on “almost every radius". In [10], [13], [18] and [26] it was proved by distinct methods that if $f \in \mathcal{D}=B^{2}$ then

$$
\left|f^{\prime}\left(r e^{i \theta}\right)\right|=o\left(\frac{1}{(1-r)^{1 / 2}}\right), \quad \text { as } r \rightarrow 1, \text { for almost every } \theta \in[0,2 \pi] .
$$

Hallenbeck and Samotij [18] proved that this estimate is sharp in a very strong sense. Indeed, they proved the following result.

Theorem B. Let $\varepsilon:(0,1) \rightarrow \mathbb{R}$ be a positive function such that $\varepsilon(r) \rightarrow 0$, as $r \rightarrow 1$. Then there exists a function $g \in \mathcal{D}$ for which

$$
\limsup _{r \rightarrow 1}\left[\frac{(1-r)^{1 / 2} \min _{|z|=r}\left|g^{\prime}(z)\right|}{\varepsilon(r)}\right]=\infty .
$$

Our main result is this paper is the following extension of the above results to all $B^{p}$ spaces, $1<p<\infty$.

Theorem 1.1. (i) Suppose that $1<p<\infty$ and $f \in B^{p}$. Then

$$
\left|f^{\prime}\left(r e^{i \theta}\right)\right|=\mathrm{o}\left(\frac{1}{(1-r)^{1-\frac{1}{p}}}\right), \quad \text { as } r \rightarrow 1, \text { for almost every } \theta \in[0,2 \pi] .
$$

(ii) Suppose that $1<p<\infty$ and let $\varepsilon:(0,1) \rightarrow \mathbb{R}$ be a positive function such that $\varepsilon(r) \rightarrow 0$, as $r \rightarrow 1$. Then there exists a function $g \in B^{p} \cap H^{\infty}$, given by a power series with Hadamard gaps, for which

$$
\limsup _{r \rightarrow 1}\left[\frac{(1-r)^{1-\frac{1}{p}} \min _{|z|=r}\left|g^{\prime}(z)\right|}{\epsilon(r)}\right]=\infty .
$$

Section 2 will contain the proof of Theorem 1.1 and we will apply this result to the study of pointwise multipliers from $B^{p}$ into $B^{q}(1<q<p<\infty)$ in Section 3. In Section 4 we will present other results in the spirit of Theorem 1.1 (ii), showing the sharpness of (1.2) and (1.3) in a way different from that in Theorem A.

Let us finish this section mentioning that, as usual, throughout the paper we shall be using the convention that $C=C(p, \alpha, q, \beta, \ldots)$ will denote a positive constant which depends only upon the displayed parameters $p, \alpha, q, \beta \ldots$ (which most of the times will be omitted) but not necessarily the same at different occurrences. Moreover, for two real-valued functions $E_{1}, E_{2}$ we write $E_{1} \lesssim E_{2}$, or $E_{1} \gtrsim E_{2}$, if there exists a positive constant $C$ independent of the arguments such that $E_{1} \leq C E_{2}$, respectively $E_{1} \geq C E_{2}$. If we have $E_{1} \lesssim E_{2}$ and $E_{1} \gtrsim E_{2}$ simultaneously then we say that $E_{1}$ and $E_{2}$ are equivalent and we write $E_{1} \asymp E_{2}$. Also, if $1<p<\infty$, $p^{\prime}$ will stand for its conjugate exponent, that is, $\frac{1}{p}+\frac{1}{p^{\prime}}=1$. 


\section{Proof of Theorem 1.1}

Proof of Theorem 1.1(i). Take $p \in(1, \infty)$ and $f \in B^{p}$. A well known result of Hardy and Littlewood [19] (see also, e. g., [8, Theorem 5.6$]$, [11, Theorem 6], or [30, Theorem 4. 28]) shows that

$$
\int_{\mathbb{D}}\left(1-|z|^{2}\right)^{2 p-2}\left|f^{\prime \prime}(z)\right|^{p} d A(z)<\infty
$$

and then it follows that

$$
\int_{0}^{1}(1-\rho)^{2 p-2}\left|f^{\prime \prime}\left(\rho e^{i \theta}\right)\right|^{p} d \rho<\infty,
$$

for almost every $\theta \in[0,2 \pi]$. Take $\theta$ for which (2.1) is true and $\epsilon>0$. Then there exists $r_{0} \in(0,1)$ such that

$$
\int_{r_{0}}^{1}(1-r)^{2 p-2}\left|f^{\prime \prime}\left(\rho e^{i \theta}\right)\right|^{p} d \rho<\epsilon^{p}
$$

We have

$$
\int_{0}^{r}\left|f^{\prime \prime}\left(\rho e^{i \theta}\right)\right| d \rho=\int_{0}^{r_{0}}\left|f^{\prime \prime}\left(\rho e^{i \theta}\right)\right| d \rho+\int_{r_{0}}^{r}\left|f^{\prime \prime}\left(\rho e^{i \theta}\right)\right| d \rho, \quad r_{0}<r<1 .
$$

Using Hölder's inequality, we see that for $r_{0}<r<1$,

$$
\begin{aligned}
\int_{r_{0}}^{r}\left|f^{\prime \prime}\left(\rho e^{i \theta}\right)\right| d \rho & =\int_{r_{0}}^{r}(1-\rho)^{2-\frac{2}{p}}\left|f^{\prime \prime}\left(\rho e^{i \theta}\right)\right|(1-\rho)^{\frac{2}{p}-2} d \rho \\
& \leq\left(\int_{r_{0}}^{r}(1-\rho)^{2 p-2}\left|f^{\prime \prime}\left(\rho e^{i \theta}\right)\right|^{p} d \rho\right)^{\frac{1}{p}}\left(\int_{r_{0}}^{r}(1-\rho)^{2\left(\frac{1}{p}-1\right) p^{\prime}} d \rho\right)^{\frac{1}{p^{\prime}}} \\
& \leq \epsilon\left(\int_{0}^{r}(1-\rho)^{-2}\right)^{1 / p^{\prime}} \\
& \leq \frac{\epsilon}{(1-r)^{1 / p^{\prime}}} \\
& =\frac{\epsilon}{(1-r)^{1-\frac{1}{p}}} .
\end{aligned}
$$

This and (2.2) give

$$
(1-r)^{1-\frac{1}{p}} \int_{0}^{r}\left|f^{\prime \prime}\left(\rho e^{i \theta}\right)\right| d \rho=\mathrm{o}(1), \quad \text { as } r \rightarrow 1,
$$

a fact, which given that

$$
\left|f^{\prime}\left(r e^{i \theta}\right)\right| \leq\left|f^{\prime}(0)\right|+\int_{0}^{r}\left|f^{\prime \prime}\left(\rho e^{i \theta}\right)\right| d \rho
$$

implies

$$
\left|f^{\prime}\left(r e^{i \theta}\right)\right|=o\left((1-r)^{\frac{1}{p}-1}\right) \quad \text { as } r \rightarrow 1 .
$$

Now we turn to prove part (ii) of our theorem. This is the extension of Theorem $\mathrm{B}$ to all $B^{p}$ spaces. Our proof is different from the given for $p=2$ in [18] and we believe that it is somewhat simpler. We shall use the following result which is the Lemma in p. 339 of [23]. 
Lemma C. Let $S_{k}$ be a sequence of positive numbers such that $\frac{S_{k}}{S_{k+1}} \rightarrow 0$, as $k \rightarrow \infty$. Then

$$
\sum_{j=1}^{k-1} S_{j}=\mathrm{o}\left(S_{k}\right), \quad \text { as } k \rightarrow \infty
$$

and

$$
\sum_{j=k+1}^{\infty} S_{j}^{-1}=\mathrm{o}\left(S_{k}^{-1}\right), \quad \text { as } k \rightarrow \infty .
$$

Proof of Theorem 1.1(ii). Set

$$
\eta(r)=\sqrt{\epsilon(r)}, \quad 0<r<1 .
$$

Clearly, it is enough to prove that there exist $g \in B^{p}$, a constant $C>0$, and an increasing sequence $\left\{r_{k}\right\}_{k=1}^{\infty} \subset$ $(0,1)$, with $\lim \left\{r_{k}\right\}=1$, for which

$$
\limsup _{k \rightarrow \infty} \frac{\left(1-r_{k}\right)^{1-\frac{1}{p}} \min _{|z|=r_{k}}\left|g^{\prime}(z)\right|}{\eta\left(r_{k}\right)} \geq C .
$$

Take a sequence $\left\{\lambda_{n}\right\}_{n=1}^{\infty}$ of positive integers satisfying the following two conditions

$$
\begin{aligned}
& \text { (a) } \frac{\lambda_{n+1}}{\lambda_{n}} \rightarrow \infty \text {, as } n \rightarrow \infty . \\
& \text { (b) } \eta\left(1-\frac{1}{\lambda_{n}}\right) \leq \frac{1}{n} \text { for all } n .
\end{aligned}
$$

Define

$$
a_{n}=\frac{\lambda_{n}^{-1 / p}}{n}, \quad n=1,2,3, \ldots
$$

and

$$
g(z)=\sum_{n=1}^{\infty} a_{n} z^{\lambda_{n}}, \quad z \in \mathbb{D} .
$$

Observe that

$$
\sum_{n=1}^{\infty} \lambda_{n}\left|a_{n}\right|^{p}=\sum_{n=1}^{\infty} \frac{1}{n^{p}}<\infty .
$$

We have that $g$ is an analytic function in $\mathbb{D}$ which is given by a power series with Hadamard gaps (by (a)). Then (2.4) implies that $g \in B^{p}$ (see, e. g., [6, p. 55]). Also, (a) implies that $\sum_{n=1}^{\infty}\left|a_{n}\right|<\infty$ and, hence, $g \in H^{\infty}$. Consequently,

$$
g \in B^{p} \cap H^{\infty} .
$$

Define also

$$
r_{k}=1-\frac{1}{\lambda_{k}}, \quad k=1,2,3, \ldots
$$

For $|z|=r_{k}$, we have

$$
\begin{aligned}
\left|g^{\prime}(z)\right| & \geq\left|z g^{\prime}(z)\right| \\
& =\left|\sum_{n=1}^{\infty} \frac{\lambda_{n}^{1-\frac{1}{p}}}{n} z^{\lambda_{n}}\right| \\
& \geq A_{k}-B_{k}-C_{k} .
\end{aligned}
$$

where, 


$$
\begin{aligned}
A_{k} & =\frac{\lambda_{k}^{1-\frac{1}{p}}}{k} \cdot\left(1-\frac{1}{\lambda_{k}}\right)^{\lambda_{k}}, \\
B_{k} & \leq \sum_{n=1}^{k-1} \frac{\lambda_{n}^{1-\frac{1}{p}}}{n}, \\
C_{k} & \leq \sum_{n=k+1}^{\infty} \frac{\lambda_{n}^{1-\frac{1}{p}}}{n} r_{k}^{\lambda_{n}} .
\end{aligned}
$$

We have

$$
A_{k}=\frac{\lambda_{k}^{1-\frac{1}{p}}}{k}\left(1-\frac{1}{\lambda_{k}}\right)^{\lambda_{k}} \geq e^{-2} \frac{\lambda_{k}^{1-\frac{1}{p}}}{k} .
$$

If we set $\alpha_{j}=\lambda_{j}^{1-\frac{1}{p}} \frac{1}{j}$, using (a), we see that

$$
\frac{\alpha_{j}}{\alpha_{j+1}}=\frac{j+1}{j}\left(\frac{\lambda_{j}}{\lambda_{j+1}}\right)^{1-\frac{1}{p}} \rightarrow 0, \quad \text { as } j \rightarrow \infty .
$$

Then, using Lemma C, we obtain

$$
B_{k}=0\left(\frac{\lambda_{k}^{1-\frac{1}{p}}}{k}\right)
$$

Defining now $\beta_{j}^{-1}=\frac{\lambda_{j}^{1-\frac{1}{p}} r_{k}^{\lambda_{j}}}{j}$, we have that

$$
\frac{\beta_{j}}{\beta_{j+1}}=\frac{j}{j+1}\left(\frac{\lambda_{j+1}}{\lambda_{j}}\right)^{1-\frac{1}{p}} r_{k}^{\lambda_{j+1}-\lambda_{j}} \leq \lambda_{j+1}^{1-\frac{1}{p}} r_{k}^{\frac{\lambda_{j+1}}{2}} \rightarrow 0, \quad \text { as } j \rightarrow \infty .
$$

Using again Lemma $\mathrm{C}$, it follows that

$$
C_{k}=o\left(\frac{\lambda_{k}^{1-\frac{1}{p}} r_{k}^{\lambda_{k}}}{k}\right)=o\left(\frac{\lambda_{k}^{1-\frac{1}{p}}}{k}\right) .
$$

Using (2.5), (2.6) and (2.7), the definitions of the sequences $\left\{\lambda_{j}\right\}$ and $\left\{r_{j}\right\}$, and (b), we deduce that there exists a constant $C>0$ such that, if $|z|=r_{k}$ and $k$ is big enough, then

$$
\left|g^{\prime}(z)\right| \geq C \frac{\lambda_{k}^{1-\frac{1}{p}}}{k}=C \frac{\left(1-r_{k}\right)^{\frac{1}{p}-1}}{k} \geq C\left(1-r_{k}\right)^{\frac{1}{p}-1} \eta\left(r_{k}\right) .
$$

This gives (2.3), finishing the proof.

Let us remark that Hallenbeck y Samotij in their work [18] actually studied the radial growth of the successive derivatives $f^{(k)}(k \in \mathbb{N})$ of functions $f$ in the Dirichlet space. The arguments that we have used to prove Theorem 1.1 can be adapted to extend it to all $B^{p}$ spaces. Namely, we can prove the following result.

Theorem 2.1. Suppose that $1<p<\infty$ and let $k$ be a positive integer.

(i) If $f \in B^{p}$, then

$$
\left|f^{(k)}\left(r e^{i \theta}\right)\right|=o\left(\frac{1}{(1-r)^{k-\frac{1}{p}}}\right), \quad \text { for almost every } \theta \in[0,2 \pi] .
$$

(ii) Let $\varepsilon:(0,1) \rightarrow \mathbb{R}$ be a positive function such that $\varepsilon(r) \rightarrow 0$, as $r \rightarrow 1$. Then there exists a function $g \in B^{p}$ for which,

$$
\limsup _{r \rightarrow 1} \frac{(1-r)^{k-\frac{1}{p}} \min _{|z|=r}\left|g^{(k)}(z)\right|}{\epsilon(r)}=\infty .
$$


We omit the details of the proof.

\section{An application of Theorem 1.1 to multipliers}

For $g \in \mathcal{H}$ ol $(\mathbb{D})$, the multiplication operator $M_{g}$ is defined by

$$
M_{g}(f)(z) \stackrel{\text { def }}{=} g(z) f(z), \quad f \in \mathcal{H} \operatorname{col}(\mathbb{D}), \quad z \in \mathbb{D} .
$$

If $X$ and $Y$ are two spaces of analytic function in $\mathbb{D}$ (which will always be assumed to be Banach or $F$-spaces continuously embedded in $\mathcal{H} o l(\mathbb{D}))$ and $g \in \mathcal{H}$ ol $(\mathbb{D})$ then $g$ is said to be a (pointwise) multiplier from $X$ to $Y$ if $M_{g}(X) \subset Y$. The space of all multipliers from $X$ to $Y$ will be denoted by $M(X, Y)$ and $M(X)$ will stand for $M(X, X)$. Using the closed graph theorem we see that if $g \in M(X, Y)$ then $M_{g}$ is a bounded operator from $X$ into $Y$.

It is well known that if $X$ is nontrivial then $M(X) \subset H^{\infty}$ (see, e. g., [1, Lemma 1.1] or [27, Lemma 1. 10]). Clearly, this implies the following:

$$
\text { If } Y \text { is nontrivial and } Y \subset X \text { then } M(X, Y) \subset H^{\infty} \text {. }
$$

The spaces of multipliers $M\left(B^{p}, B^{q}\right)$ have been studied in a good number of papers (see $[4,12,15,16,27$, $28,31]$ and the references therein). In particular, the following result holds.

Theorem D. If $1 \leq q<p<\infty$ then $M\left(B^{p}, B^{q}\right)=\{0\}$.

This was proved in [12] using, among other facts, a decomposition theorem for Besov spaces and Khinchine's inequality. The authors of this paper gave in [5] a new proof using Theorem A. Next we are going to give a new proof Theroem D using Theorem 1.1.

Proof of Theorem $D$. Since $B^{1} \subset B^{q}$ for all $q>1$, it suffices to consider the case $1<q<p<\infty$. So, assume this and take $g \in M\left(B^{p}, B^{q}\right)$. Since the constant function 1 belongs to $B^{p}$, we see that $g \in B^{q}$. Also, the inclusion $B^{q} \subset B^{p}$ and (3.1) implies that $g \in H^{\infty}$. Thus, we have

$$
g \in B^{q} \cap H^{\infty} .
$$

Use Theorem 1.1 (ii) to pick a function $f \in B^{p} \cap H^{\infty}$, given by a power series with Hadamard gaps, with

$$
\limsup _{r \rightarrow 1}\left[(1-r)^{1-\frac{1}{q}} \min _{|z|=r}\left|f^{\prime}(z)\right|\right]=\infty \text {. }
$$

Since $M_{g}\left(B^{p}\right) \subset B^{q}$, we have that $M_{g}(f)=g \cdot f \in B^{q}$, that is,

$$
\int_{\mathbb{D}}\left(1-|z|^{2}\right)^{q-2}\left|g^{\prime}(z) f(z)+g(z) f^{\prime}(z)\right|^{q} d A(z)<\infty .
$$

Since $g \in B^{q}$ and $f \in H^{\infty}$ we see that

$$
\int_{\mathbb{D}}\left(1-|z|^{2}\right)^{q-2}\left|g^{\prime}(z) f(z)\right|^{q} d A(z)<\infty .
$$

This and (3.3) imply that

$$
\int_{\mathbb{D}}\left(1-|z|^{2}\right)^{q-2}\left|g(z) f^{\prime}(z)\right|^{q} d A(z)<\infty,
$$


or, equivalently,

$$
\int_{0}^{1}(1-r)^{q-2} M_{q}\left(r, g f^{\prime}\right)^{q} d r<\infty .
$$

We will show that $g \equiv 0$ arguing by contradiction. So, assume that $g \not \equiv 0$. Since $g \in H^{\infty}$ and $g \not \equiv 0$, using Fatou's theorem and the Riesz uniqueness theorem [8], we see that $g$ has a finite non-zero radial limit at almost every point $e^{i \theta}$ of $\partial \mathbb{D}$. Then it follows that there exist $C>0, r_{0} \in(0,1)$, and a measurable set $E \subset[0,2 \pi]$ whose Lebesgue measure $|E|$ is positive such that

$$
\left|g\left(r e^{i \theta}\right)\right| \geq C, \quad r_{0}<r<1, \quad \theta \in E .
$$

This and (3.4) readily imply that

$$
\int_{0}^{1} \int_{E}(1-r)^{q-2}\left|f^{\prime}\left(r e^{i \theta}\right)\right|^{q} d \theta d r<\infty .
$$

Since $f$ is given by a power series with Hadamard gaps, using an extension of Lemma 6.5 in [32, Vol. 1, p. 203] (see [17, Lemma 1]), it follows that

$$
\int_{E}\left|f^{\prime}\left(r e^{i \theta}\right)\right|^{q} d \theta \asymp M_{q}\left(r, f^{\prime}\right)^{q} .
$$

This and (3.5) yield that $f \in B^{q}$. Using Theorem 1.1(i), we see that this in contradiction (3.2). This finishes the proof.

\section{Further results}

This section is devoted to prove another result in the line of those in Theorem A and in Theorem 1.1(ii).

Theorem 4.1. Suppose that $1<p<\infty$ and let $\phi$ be a positive and increasing function defined in $[0,1)$ with

$$
\int_{0}^{1}(1-r)^{p-2} \phi(r)^{p} d r<\infty .
$$

Then there exists a function $f \in B^{p} \cap H^{\infty}$ such that

$$
\limsup _{r \rightarrow 1^{-}} \frac{\min \left|f^{\prime}(z)\right|}{\phi(r)}=\infty .
$$

Lemma $\mathrm{C}$ and the following one will be used in our proof of Theorem 4.1

Lemma 4.2. Suppose that $1<p<\infty$ and let $\phi$ be a positive and increasing function defined in $(0,1)$ and satisfying (4.1). Then there exist another positive and increasing function $\phi_{1}$, defined in $(0,1)$ satisfying

$$
\int_{0}^{1}(1-r)^{p-2} \phi_{1}(r) d r<\infty \text { and } \lim _{r \rightarrow 1^{-}} \frac{\phi_{1}(r)}{\phi(r)}=\infty .
$$

Proof. For $k=0,1,2, \ldots$, set

$$
r_{k}=1-2^{-k}, \quad a_{k}=\int_{r_{k}}^{r_{k+1}}(1-r)^{p-2} \phi(r)^{p} d r .
$$


Clearly, (4.1) implies that $\sum_{k=0}^{\infty} a_{k}<\infty$. Now, use a well known theorem of Dini (see [24, p. 293]), to pick an increasing sequence of positive numbers $\left\{c_{k}\right\}_{k=0}^{\infty}$ with

$$
\lim \left\{c_{k}\right\}=\infty \text { and } \sum_{k=0}^{\infty} a_{k} c_{k}<\infty
$$

It is easy to see that the function $\phi_{1}$ defined by

$$
\phi_{1}(r)=c_{k}^{1 / p} \phi(r), \quad r \in\left[r_{k}, r_{k+1}\right), \quad k=0,1,2, \ldots,
$$

satisfies the conclusion of the lemma.

Proof of Theorem 4.1. It is clear that we may assume without loss of generality that $\phi(r) \rightarrow \infty$, as $r \rightarrow 1$ and, bearing in mind Lemma 4.2, it suffices to prove that there exist $f \in B^{p}$ and a constact $C>0$ such that

$$
\limsup _{r \rightarrow 1^{-}} \frac{\min _{|z|=r}\left|f^{\prime}(z)\right|}{\phi(r)}>C .
$$

Take a sequence $\left\{r_{k}\right\}_{k=1}^{\infty} \subset(0,1)$ with the following properties:

(a) $\left\{r_{k}\right\}$ is strictly increasing and $\lim \left\{r_{k}\right\}=1$.

(b) $r_{k+1}-r_{k}>\frac{1}{2}\left(1-r_{k}\right)$, for every $k$.

(c) $\left(1-r_{k+1}\right)^{1+\frac{1}{p}} \cdot\left(1-r_{k}\right)^{-2} \rightarrow 0$, as $k \rightarrow \infty$.

(d) $\frac{\phi\left(r_{k+1}\right)}{\phi\left(r_{k}\right)} \rightarrow \infty$, as $k \rightarrow \infty$.

Note that (b) implies

$$
1-r_{k}=\left(1-r_{k+1}\right)+\left(r_{k+1}-r_{k}\right)>\left(1-r_{k+1}\right)+\frac{1}{2}\left(1-r_{k}\right)
$$

and, hence,

$$
\frac{1-r_{k}}{1-r_{k+1}}>2 \text { or, equivalently, } 1-r_{k+1}<\frac{1}{2}\left(1-r_{k}\right)
$$

This gives

$$
\left(1-r_{k+1}\right)^{p-1}<\frac{1}{2^{p-1}}\left(1-r_{k}\right)^{p-1}
$$

and then we see that

$$
\frac{1}{p-1}\left(\left(1-r_{k}\right)^{p-1}-\left(1-r_{k+1}\right)^{p-1}\right) \geq A\left(1-r_{k}\right)^{p-1},
$$

with $A=\frac{1}{p-1}\left(1-\frac{1}{2^{p-1}}\right)$.

Using the fact that $\phi$ is increasing and (4.4), we obtain

$$
\begin{aligned}
\infty & >\sum_{k=1}^{\infty} \int_{r_{k}}^{r_{k+1}}(1-r)^{p-2} \phi(r)^{p} d r \\
& \geq \sum_{k=1}^{\infty} \phi\left(r_{k}\right)^{p} \int_{r_{k}}^{r_{k+1}}(1-r)^{p-2} d r \\
& =\sum_{k=1}^{\infty} \phi\left(r_{k}\right)^{p} \frac{\left(1-r_{k}\right)^{p-1}-\left(1-r_{k+1}\right)^{p-1}}{p-1} \\
& \geq A \sum_{k=1}^{\infty} \phi\left(r_{k}\right)^{p}\left(1-r_{k}\right)^{p-1} .
\end{aligned}
$$


Then we have

$$
\sum_{k=1}^{\infty} \phi\left(r_{k}\right)^{p}\left(1-r_{k}\right)^{p-1}<\infty
$$

Define now

$$
n_{k}=\left[\frac{1}{1-r_{k}}\right], \quad k \geq 1
$$

Here, as usual, for $x \in \mathbb{R},[x]$ denotes the integral part of $x$. Define also

$$
a_{k}=\phi\left(r_{k}\right)\left(1-r_{k}\right), \quad k=1,2, \ldots
$$

and

$$
f(z)=\sum_{k=1}^{\infty} a_{k} z^{n_{k}}, \quad z \in \mathbb{D} .
$$

Bearing in mind the definition of the sequence $\left\{n_{k}\right\}$ and (4.3), we see that $f$ is an analytic function in $\mathbb{D}$ which is given by a power series with Hadamard gaps. Furthermore,

$$
\sum_{k=1}^{\infty} n_{k}\left|a_{k}\right|^{p} \approx \sum_{k=1}^{\infty}\left(1-r_{k}\right)^{p-1} \phi\left(r_{k}\right)^{p}<\infty .
$$

Then, we see that $f \in B^{p}$.

Using Hölder's inequality, (4.5), (4.3), and the definition of the sequence $\left\{n_{k}\right\}$, we see also that

$$
\sum_{k=1}^{\infty}\left|a_{k}\right| \leq\left(\sum_{k=1}^{\infty} n_{k}\left|a_{k}\right|^{p}\right)^{1 / p}\left(\sum_{k=1}^{\infty} \frac{1}{n_{k}^{p^{\prime} / p}}\right)^{1 / p^{\prime}}<\infty,
$$

which implies that $f \in H^{\infty}$. Consequently,

$$
f \in B^{p} \cap H^{\infty}
$$

Now, for $|z|=r_{k}$, we have

$$
\begin{aligned}
\left|f^{\prime}(z)\right| & \geq\left|z f^{\prime}(z)\right| \\
& =\left|\sum_{j=1}^{\infty} n_{j} \phi\left(r_{j}\right)\left(1-r_{j}\right) z^{n_{j}}\right| \\
& \geq n_{k} \phi\left(r_{k}\right)\left(1-r_{k}\right) r_{k}^{n_{k}}-\sum_{j=1}^{k-1} n_{j} \phi\left(r_{j}\right)\left(1-r_{j}\right)-\sum_{j=k+1}^{\infty} n_{j} \phi\left(r_{j}\right)\left(1-r_{j}\right) r_{k}^{n_{j}} \\
& =I-I I-I I I .
\end{aligned}
$$

Then it follows that

$$
\min _{|z|=r_{k}}\left|f^{\prime}(z)\right| \geq I-I I-I I I .
$$

Bearing in mind the definition of $n_{k}$, it is clear that there exists $C>0$ such that

$$
I \geq C \phi\left(r_{k}\right)
$$

Using again the definition of the sequence of exponents $\left\{n_{j}\right\}$ and property (d), we see that

$$
\frac{n_{j}\left(1-r_{j}\right) \phi\left(r_{j}\right)}{n_{j+1}\left(1-r_{j+1}\right) \phi\left(r_{j+1}\right)} \approx \frac{\phi\left(r_{j}\right)}{\phi\left(r_{j+1}\right)} \rightarrow 0, \quad \text { as } j \rightarrow \infty
$$

and then Lemma $\mathrm{C}$ implies that

$$
I I=\sum_{j=1}^{k-1} n_{j} \phi\left(r_{j}\right)\left(1-r_{j}\right) r_{k}^{n_{j}}=\mathrm{o}\left(\phi\left(r_{k}\right)\right)
$$


Let us turn to estimate $I I I$. Using the definition of the $n_{j}$ 's and the inequality $(1-x)^{n}<2(n x)^{-2}$, which holds for $n>0$ and $0<x<1$, we deduce that

$$
\begin{aligned}
I I I & =\sum_{j=k+1}^{\infty} n_{j} \phi\left(r_{j}\right)\left(1-r_{j}\right) r_{k}^{n_{j}} \\
& \approx \sum_{j=k+1}^{\infty} \phi\left(r_{j}\right)\left(1-\frac{1}{n_{k}}\right)^{n_{j}} \\
& \leq 2 n_{k}^{2} \sum_{j=k+1}^{\infty} \frac{\phi\left(r_{j}\right)}{n_{j}^{2}} .
\end{aligned}
$$

Since $\phi$ is increasing and satisfies (4.1) we see that

$$
\phi(r)=o\left(\frac{1}{(1-r)^{1-\frac{1}{p}}}\right) .
$$

Then, using (c), we deduce that

$$
\begin{aligned}
\frac{n_{j}^{2} / \phi\left(r_{j}\right)}{n_{j+1}^{2} / \phi\left(r_{j+1}\right)} & \approx \frac{\left(1-r_{j+1}\right)^{2} \phi\left(r_{j+1}\right)}{\left.\left(1-r_{j}\right)^{2}\right) \phi\left(r_{j}\right)} \\
& =\frac{\left(1-r_{j+1}\right)^{1+\frac{1}{p}}}{\left(1-r_{j}\right)^{2}} \cdot \frac{\left(1-r_{j+1}\right)^{1-\frac{1}{p}} \phi\left(r_{j+1}\right)}{\phi\left(r_{j}\right)} \\
& \lesssim \frac{\left(1-r_{j+1}\right)^{1+\frac{1}{p}}}{\left(1-r_{j}\right)^{2}} \\
& =\mathrm{o}(1) .
\end{aligned}
$$

Then, using (4.6) and Lemma C, we obtain

$$
I I I=\mathrm{o}\left(\phi\left(r_{k}\right)\right)
$$

This, together with the bounds obtained for $I$ and $I I$, implies that

$$
\liminf _{k \rightarrow \infty} \frac{\min _{|z|=r_{k}}\left|f^{\prime}(z)\right|}{\phi\left(r_{k}\right)} \geq C .
$$

This gives (4.2).

\section{References}

[1] A. Aleman, P. L. Duren, M. J. Martín and D. Vukotić, Multiplicative isometries and isometric zero-divisors, Canad. J. Math. 62 (2010), no. 5, 961-974.

[2] J. M. Anderson, J. Clunie and Ch. Pommerenke, On Bloch functions and normal functions, J. Reine Angew. Math. 270 (1974), 12-37.

[3] J. Arazy, S. D. Fisher and J. Peetre, Möbius invariant function spaces, J. Reine Angew. Math. 363 (1985), 110-145.

[4] N. Arcozzi, R. Rochberg and E. Sawyer, Carleson measures for analytic Besov spaces, Rev. Mat. Iberoamericana 18 (2002), 443-510.

[5] S. Domínguez and D. Girela, A radial integrability result concerning bounded functions in analytic Besov spaces with applications, Results in Mathematics 75, Article number 67 (2020).

[6] J. J. Donaire, D. Girela and D. Vukotić, On univalent functions in some Möbius invariant spaces, J. Reine Angew. Math. 553 (2002), 43-72.

[7] J. J. Donaire, D. Girela and D. Vukotić, On the growth and range of functions in Möbius invariant spaces, J. Anal. Math. 112 (2010), 237-260.

[8] P. L. Duren, Theory of $H^{p}$ spaces, Academic Press, New York-London, 1970. Reprint: Dover, Mineola-New York, 2000. 
[9] P. L. Duren and A. P. Schuster, Bergman Spaces, Math. Surveys and Monographs, Vol. 100, American Mathematical Society, Providence, Rhode Island, 2004.

[10] T. M. Flett, On the radial order of a univalent function, J. Math. Soc. Japan 11 (1959), 1-3.

[11] T. M. Flett, The dual of an inequality of Hardy and Littlewood and some related inequalities, J. Math. Anal. Appl. 38 (1972), 746-765.

[12] P. Galanopoulos, D. Girela and J. A. Peláez, Multipliers and integration operators on Dirichlet spaces, Trans. Amer. Math. Soc. 363 (2011), no. 4, 1855-1886.

[13] F. W. Gehring, On the radial order of subharmonic functions, J. Math. Soc. Japan 9 (1957), 77-79.

[14] D. Girela, Analytic functions of bounded mean oscillation, Complex Function Spaces, Mekrijärvi 1999. Editor: R. Aulaskari. Univ. Joensuu Dept. Math. Rep. Ser. 4, Univ. Joensuu, Joensuu (2001) pp. 61-170.

[15] D. Girela and N. Merchán, Multipliers and integration operators between conformally invariant spaces, Rev. R. Acad. Cienc. Exactas Fs. Nat. Ser. A Mat. RACSAM. 114 (2020), Article number 181.

[16] D. Girela and J. A. Peláez, Carleson measures, multipliers and integration operators for spaces of Dirichlet type, J. Funct. Analysis 241 (2006), n. 1, 334-358.

[17] D. Girela and M. M. Rodríguez, Sharp estimates on the radial growth of the derivative of bounded analytic functions, Complex Variables Theory Appl. 28 (1996), no. 3, 271-283.

[18] D. J. Hallenbeck and K. Samotij, Radial growth and variation of Dirichlet finite holomorphic functions in the disk. Colloq. Math. 58 (1990), no. 2, 317-325.

[19] G. H. Hardy and J. E. Littlewood, Some properties of fractional integrals, II, Math. Z. 34 (1932), 403-439.

[20] H. Hedenmalm, B. Korenblum and K. Zhu, Theory of Bergman Spaces, Graduate Texts in Mathematics 199, Springer, New York, Berlin, etc. 2000.

[21] F. Holland and D. Walsh, Growth estimates for functions in the Besov spaces $A_{p}$, Proc. Roy. Irish Acad. Sect. A 88 (1988), 1-18.

[22] F. Holland and D. Walsh, Distributional inequalities for functions in Besov spaces, Proc. Roy. Irish Acad. Sect. A 94 (1994), no. 1, 1-17.

[23] P. B. Kennedy, On the derivative of a function of bounded characteristic, Quart. J. Math. Oxford Ser. (2) 15 (1964), $337-341$.

[24] K. Knopp, Theory and Application of Infinite Series, Hafner Pu. Co., New York, 1971.

[25] W. Rudin, The radial variation of analytic functions, Duke Math. J. 22 (1955), 235-242.

[26] W. Seidel and J. L. Walsh, On the derivatives of functions analytic in the unit circle and their radii of univalence and of $p$ valence, Trans. Amer. Math. Soc. 52 (1942), 128-216.

[27] S. A. Vinogradov, Multiplication and division in the space of analytic functions with area integrable derivative, and in some related spaces (in Russian), Zap. Nauchn. Sem. S.-Peterburg. Otdel. Mat. Inst. Steklov. (POMI) 222 (1995), Issled. po Linein. Oper. i Teor. Funktsii 23, 45-77, 308. English translation in J. Math. Sci. (New York) 87, no. 5 (1997), 3806-3827.

[28] Z. Wu, Carleson measures and multipliers for Dirichlet spaces. J. Funct. Anal. 169 (1999), no. 1, 148-163.

[29] K. Zhu, Analytic Besov spaces, J. Math. Anal. Appl. 157 (1991), 318-336.

[30] K. Zhu, Operator Theory in Function Spaces, Second Edition, Math. Surveys and Monographs, 138 (2007).

[31] N. Zorboska, Multiplication and Toeplitz operators on the analytic Besov spaces. In: More Progress in Analysis: Proceedings of the 5th International. Isaac Congress. Catania, Italy, 25-30 July 2005. Editors: H. G. W. Begehr and F. Nicolosi. World Scientific (2009), pp. 387-396.

[32] A. Zygmund, Trigonometric Series, Vol. I and Vol. II, Second edition, Camb. Univ. Press, Cambridge, 1959. 